\title{
Alegoría, mito y artes liberales en san Agustín De ordine I. 8. 24
}

\section{Claudio César Calabrese}

(Universidad Panamericana, Campus Aguascalientes, Departamento de Humanidades)

\section{Allegory, Myth and Liberal Arts in St. Augustine De ordine I. 8. 24}

\begin{abstract}
In this article we intend to establish a relationship between myth and allegory according to the treatment of the Hellenistic-Christian literary tradition, to consider the application of allegory with philosophical function and its reception by St. Augustine. We exemplify the process with the Augustinian interpretation of the story of Pyramus and Thisbe according to De Ordine. We conclude in the argumentative scope of the allegory to found a philosophical prescriptive.
\end{abstract}

\section{Keywords}

Saint Augustine; allegory; order; liberal arts; hermeneutics 


\section{Introducción}

En el presente artículo nos proponemos estudiar el pasaje de De ordine (I. 8. 24), en el que san Agustín, por un lado, recomienda a su discípulo Licencio, la frecuentación de la poesía para una más profunda comprensión filosófica del orden y, por otro lado, sugiere a este mismo discípulo, de marcada vocación literaria, una determinada preceptiva para releer y reescribir en clave cristiana la historia de Píramo y Tisbe, leyenda oriental, que tomó forma literaria con Ovidio (Metamorfosis IV, 55-166). En ese punto se trata la función de la alegoría.

El término "alegoría” designó un método para comprender más adecuadamente a Homero en Alejandría y se transformó, ya en Palestina, como un modo de establecer la comprensión profunda del Antiguo Testamento a partir de la figura de Cristo (Herren 2017); la llamada "interpretación alegórica" se afianzó como un modo de exégesis empeñado en descubrir un sentido más allá del literal, aunque eche raíces en él. ${ }^{1}$ Cuando estudiamos a San Agustín, la interpretación en clave alegórica de Virgilio constituye el nexo viviente de esta tradición, especialmente a través de las figuras de Servio y de Macrobio, destacados comentadores del Mantuano (Norden 1916: p. 29).

Entender los textos literarios en un sentido diverso del literal, considerando las palabras no por lo que sí dicen, sino por "decir otras cosas" (allegorein, en latín alia oratio), es procedimiento conocido y practicado desde los comienzos de la literatura griega. ${ }^{2}$ Como ya mencionamos, un modo de leer se vinculó indisolublemente a un modo de escribir, dando lugar a una literatura deliberadamente alegórica; una aproximación a este proceso nos pone necesariamente en estrecha relación con el mundo complejo y lábil del símbolo.

Desde esta perspectiva consideramos nuestro aporte: si en la concepción ovidiana de Píramo y Tisbe, el amor puede sobrevivir de manera deletérea a la muerte, a condición de que ponga todo su ser en juego, la relectura agustiniana afronta la paradoja de cómo puede brotar, de la negación implícita en la historia, la propuesta de salvación. En este punto precisamente se nos presenta el problema, a la vez filosófico y literario, en cuanto resulta la parte no delimitable de una nueva paráfrasis; en efecto, el modelo que san Agustín propone implica el paso de la poesía clásica al impulso alegórico conducido por la gracia. En razón de ello, la advertencia agustiniana de que la santidad puede darse sin estudio ni poesía, expresa también -y esto es lo más significativo para nosotros- que ambas brotan de la misma esfera de libertad y belleza del alma.

1 Si bien la metodología del trabajo requiere de una cierta precisión histórica, consideramos que no ha perdido vigencia la profunda intuición de Lewis (2013: pp. 55-57) acerca de la imposibilidad de establecer un origen en sentido estricto, pues la capacidad de la alegoría, que nuestro autor define como la representación de lo inmaterial en términos pictóricos, es natural al lenguaje y al pensamiento humanos.

2 Pfeiffer (1968: pp. 10-11, 35). Fue un recurso de los rapsodas que no solo recitaban a Homero sino que también lo interpretaban; Platón, Aristóteles y los eruditos alejandrinos rechazaron este método, pero los Estoicos lo adoptaron, legitimaron y perpetuaron. Los representantes de lengua griega del Neoplatonismo lo adoptaron como un método de apropiación de la mitología para el discurso filosófico. 
Trabajar el mencionado pasaje del De ordine implicará los siguientes pasos: a) presentar las nociones de mito y de alegoría y la relación de la última con el primero; b) tratar la recepción alegórico moralizante de mitos griegos en Horacio; c) considerar la depuración del mito como herramienta de la filosofía, según el neoplatónico Macrobio; d) presentar el texto de De ordine; e) concluir.

\section{a) Entre símbolo y alegoría}

Estudiar el surgimiento del concepto de símbolo implica realizar una historia de la cultura clásica y de cómo esta, fusionada con el cristianismo, se prolongó en la Edad Media. Evidentemente no se trata de un cúmulo narrativo de aventuras; por el contrario, se trata esencialmente, de un drama (Esparza Urzúa 2017: p. 127). La realización de una tal historia debería abarcar el estudio de la exégesis alegórica, pues de ella fueron objeto los poetas desde Homero en adelante.

Siempre que nos acercamos a la irradiación del mito asistimos a la apertura de símbolos que conllevan conocimientos del ser. Ahora bien: ¿es posible interpretar aquello que se presenta como ilimitado? La pregunta es pertinente, en cuanto el lenguaje se sostiene en la equivocidad, es decir, sobre la lógica del sentido, que resulta posible cada vez que ha sido determinada la significación; esto es lo que Ricoeur (1965: p. 22) ha denominado "estructura intencional de segundo grado".

Para los intereses de nuestro artículo, vinculamos el concepto de límite fundamentalmente con el campo semántico "saber", aunque también demarcamos el horizonte de indeterminación sobre el que se sostiene. En este contexto "saber" comienza presentándose como "poner límites a algo"; en efecto, "definir" es uno de los elementos de la inteligibilidad y, por ello, resulta significativo anotar, como su complemento, que las formas verbales "indefinidas" ponen énfasis en el aspecto en que está inmersa la acción verbal (recordemos que, en griego, el aoristo, palabra compuesta por una "a" privativa y horizo, "limitar", presenta la acción sin referencia a su duración): no es posible porque no hay límites o, con el vocabulario aristotélico de la Física (III, 6, 2007a 15), carece de cumplimiento (atelès).

En nuestro caso, establecer el límite de la interpretación implica registrar la demarcación del símbolo en alegoría. Siguiendo a Ricoeur (1965: p. 31) señalamos que limitar la interpretación significa que ha sido fijada en un concepto o, más genéricamente, conceptualizada; por ello, distinguir la comprensión de la interpretación significa que esta última tiene la capacidad de actualizar el contexto de la comprensión, volviéndola (parcialmente al menos) inadecuada.

En razón de ello, distinguimos la alegoría clásica de la cristiana (tanto tardo antigua como medieval), pues esta última se concentra plenamente en la escritura y tiene las siguientes características: "a one-to-one correspondence between each character and some concept, abstract principle, or element of the physical world” (Struck 2004: p. 3). Concordamos con el autor en que la alegoría es, ante todo, un fenómeno de lectura, fundado en la autoridad del texto interpretado. Aunque se trata de una lectura que 
considera al texto como expresión de una estructura profunda del cosmos, tal crítica literaria descansa sobre dos fundamentos distintos pero complementarios: por un lado, como ya señalamos, la interpretación de textos existentes y, por otro, la referencia a la preceptiva pensada para la obra todavía no escrita. Ciertamente no se trata de ubicar a la alegoría en el campo literario como perspectiva única, ya que ella se encuentra inmersa en la profundidad de la vida de la cultura y experimenta los cambios y transformaciones que son propias de la historia (Lamberton 1986). Con el afianzamiento de la interpretación de cuño neoplatónico, especialmente con Macrobio (Setaioli 2004), la frontera entre filosofía y literatura comienza a esfumarse, encaminando su esfuerzo exegético al núcleo mítico, como ya aparecía con claridad en el propio Platón (Tim. 22 c-d; Phaed. 69 c). Desde esta perspectiva, alegoría significa descubrir un sentido que se encuentra más allá del literal, aunque eche raíces en él; se trata de un significado que no resulta inmediatamente accesible a todos. Por esta razón, alegoría es tanto lo que se establece mediante la vía de interpretación cuanto el proceso interpretativo en sí. Se advierten aquí dos aspectos centrales para nuestra investigación: a) la exigencia de interpretar el texto de tal modo que ponga en evidencia el contenido de verdad que a priori se considera que contiene y b) las transformaciones histórico-culturales requieren de un ejercicio de interpretación permanente, porque sus claves varían con dichos cambios.

Sobre el horizonte del símbolo es posible comprender el procedimiento alegórico, pues conserva la bivalencia entre significado y significante, como dos mitades cortadas de un todo (Struck 2004: p. 92). El significado más arcaico de símbolo marca el inicio de su evolución semántica, que resulta coincidente con expresiones técnicas de la adivinación. Es posible reforzar este vínculo originario entre lenguaje de los augurios y exégesis alegórica, con la referencia a la utilización adivinatoria de los textos de Homero y de Virgilio y, en el mundo cristiano, de la Biblia.

La alegoría, entonces, es un símbolo reducido a signo, es decir, la demarcación de una de sus posibilidades dinámicas en cuanto símbolo. El signo es una expresión semiótica, la presentación convencional de algo ya conocido: la alegoría petrifica el símbolo y lo convierte en signo, aunque pueda conservar su ropaje. Para M. Eliade (1987: pp. 136138), el símbolo posee polivalencia de sentido (aspectos concretos que toma el sentido en sí), ordenando sus significados en distintos planos de la realidad.

\section{b) La recepción alegórico-moralizante de mitos griegos en Horacio}

Para aproximarnos a la experiencia literaria directa de san Agustín resulta conveniente hacer un seguimiento de textos a los que tuvo acceso en sus tiempos de gramático. En principio, se hacen presentes temas homéricos que reciben en latín una continuidad del tratamiento alegórico de origen alejandrino.

Cuando Horacio fustiga la avaricia (Sátiras I, 1, 68-72), recurre al mito: la apetencia desmedida de bienes materiales puede ser tan insaciable como la sed de Tántalo, pues contempla los bienes sin posibilidad de disfrutarlos. 
De las distintas versiones del mito, parece que Horacio parte de Homero (Od. XI, 982-992), quien describe a Tántalo de pie, en un lago; el agua le llegaba hasta la barba y, a la vera del lago o espejo de agua, con árboles cuyas ramas se encuentran encima, padece hambre y sed eternas. Cuando intenta comer los frutos, las ramas se elevan a causa del viento y cuando intenta beber, las aguas se retiran: "Tántalo, lleno de sed, intenta alcanzar el agua que rehúye sus labios... ¿De qué ríes? Con un nombre distinto, a ti se refiere la historia: por doquier acopias talegos y sobre ellos te duermes con la boca abierta". 3

La lectura alegórica de la figura mítica de Tántalo es de interés para seguir luego la propuesta agustiniana, tanto por el procedimiento cuanto por el principio según el cual la fábula, por extraordinario que pueda parecer (Quid rides?), se refiere al lector en cuanto le incumbe la enseñanza moral mentada; en efecto, el principio que aquí se establece, de te fabula narratur, puede expresarse así: los castigos que se refieren como propios del más allá se encuentran ya en esta vida; los que sufren la codicia tienen a mano lo que desean, pero no lo pueden disfrutar, como sucede con Tántalo en el Hades.

\section{c) El mito como herramienta de la filosofía según Macrobio}

A fines del siglo IV, en el marco de la filosofía neoplatónica, ${ }^{4}$ el comentarista Macrobio dedica un importante fragmento de su Commentarii in Somnium Scipionis (I, 54-57) para sostener el uso de la narración literaria (fabula) como técnica de expresión filosófica, contra el epicúreo Colotes, quien niega la eficacia de este recurso y se burla concretamente de las alegorías de Er, que encontramos en la República platónica (X, 614a-621b), y del Sueño de Escipión, que Cicerón, ${ }^{5}$ a imitación del modelo griego, había colocado al final de su propia República. ${ }^{6}$

Según Macrobio, no es razonable tener todas las ficciones en la misma consideración (nec omnibus fabulis philosophia repugnat nec omnibus adquiescit I, 57); según la perspectiva antes citada, lo propio de la filosofía será ordenarlas mediante biparticiones (diuisionum gradibus explicandum est I, 57), que delimitan sus campos de referencia. La primera distinción es entre las comedias que simplemente procuran placer estético y que, por lo tanto, no son útiles para la filosofía y aquellas que al relato suman una parénesis moral (Pepin 1976: p. 210); a estas últimas las distingue por su tema, ubicando los apólogos de Esopo por las características de sus peripecias (relationis ordo I, 57), que ilustra con ejemplos de

3 Tantalus a labris sitiens fugientia captat

flumina - quid rides? mutato nomine de te

fabula narratur: congestis undique saccis

indormis inhians...

4 Las peculiaridades filosóficas del neoplatonismo latino se encuentran cuidadosamente expuestas en Gersh (2012); Flamant (1977).

5 Stevens (2006). Sobre el conocimiento de san Agustín del Somnium cf. Bermon (2011).

6 Como atestigua san Agustín en De civitate Dei XXII, 28, el Arpinate también recreó el pasaje de Er, aunque se ha perdido hasta hoy. 
mitos religiosos de origen hesiódico u órfico-pitagórico. Rechaza como instrumento de la filosofía a aquellas en que la falsedad se encuentra tanto en el tema como en el modo de relatar (de falso per falsum narratur I, 58).

Los relatos propiamente míticos (narratio fabulosa I, 58) no son merecedores de la misma consideración, aunque todos partan de un origen verdadero; se deben desestimar de la filosofía aquellos relatos que en su ficción presentan actos inmorales, sacrilegios o adulterios entre los dioses (I, 58). Pero, cuando el conocimiento de lo sagrado se presenta mediante una ficción honesta (el viaje de Er o el sueño de Escipión), el filósofo puede recurrir a estos relatos. De esta manera, Macrobio busca distinguir la fábula del mito (a fabulis fabulosa secernere I, 58) y producir así una cualificación progresiva del mito: rechaza las fábulas totalmente ficcionales y las que expresan alguna forma de inmoralidad, para conservar los mitos que ponen de manifiesto verdad, exhortación moral y belleza en la forma literaria (Pépin 1976: p. 211).

Para Macrobio, el mito tiene valor propedéutico cuando se aplica a disertaciones sobre el alma, los demonios o los dioses inferiores, pero cuando el pensamiento se remonta a la Inteligencia o al Primer principio, el mito se muestra insuficiente. Resulta claro que Macrobio adaptó la organización y el procedimiento de las hipóstasis, pues en ellas Plotino encontró el campo de aplicación de las alegorías, aunque Macrobio prefirió el recurso de imágenes y relatos, donde los misterios se encubren y se muestran con las sinuosidades de los símbolos (sic ipsa mysteria figurarum cuniculis operiuntur I, 59).

Macrobio no precisa en qué difiere esta analogía del mito; podemos señalar que la estructura formal es casi idéntica, y que el mito -en cuanto al contenido- también puede entenderse como relato alegórico, pues la simple imagen tiene la capacidad de reflejar una experiencia determinada (Junco 2016: p. 21).

No forma parte de nuestro trabajo ingresar en los modos en que la Patrística retoma la alegoría como tradición exegética; únicamente anotamos que esta concepción, según la cual las palabras del intérprete determinan el verdadero significado de un texto, será una ocupación central de san Agustín en su disputa contra los representantes del Maniqueísmo, quienes sostuvieron una interpretación solo alegórica del Antiguo Testamento. Para san Agustín fijar el sentido literal constituye el paso imprescindible para determinar el oculto o alegórico; como en Plotino (IV, 3, 11, 6-8), la interpretación es un instrumento de análisis de las circunstancias del texto y de la didáctica de sus diversos alcances. Por lo tanto, sigue el curso interpretativo de las alegorías que, en la Antigüedad Tardía, se hizo sobre los modelos de Homero y Virgilio. Hemos seguido esta perspectiva teórica en la sistematización que sobre el tema realizó Macrobio.

\section{d) De Ordine: Artes liberales y preceptiva alegórica}

Por Las Confesiones de san Agustín conocemos su vasta cultura literaria y los círculos de intelectuales que frecuentó y a cuyo calor robusteció su pensamiento; en los diálogos, en especial el que aquí nos ocupa, De ordine, se refleja esta vasta cultura y los modos en que comienza a proyectarse como instrumento de aproximación al misterio. 
La organización del diálogo. El tema central del texto es la Providencia, cuyo tratamiento también había estado presente en dos obras de la misma época: La vida feliz y Contra académicos.

El primer paso planteado consiste en comprender el orden de la naturaleza, y se cumple en los siguientes términos: siempre en un contexto de gozo ante la concordia del conjunto de lo viviente, san Agustín reflexiona sobre los diversos modos en que está presente aquella armonía; refiere tanto a las características de su entorno natural, cuanto a la alegría de aquellos días de plenitud amical e intelectual.

Desde el inicio del diálogo, san Agustín plantea la cuestión con toda su dificultad: es, en sí mismo, problemático aclarar el orden de las cosas, el propio de cada una y el universal, pero aún más difícil resulta explicar por qué, en un mundo regido por la Providencia, existe el mal (perversitas usquequaeque diffusa est I. 1. 1); dicho planteo puede llevar a dos conclusiones, que san Agustín se apresura a considerar erróneas: o bien el gobierno de la Providencia no llega a todas las cosas (más precisamente, a las inferiores en la escala del ser, in haec ultima I. 1. 1) o bien que los males ocurren por voluntad de Dios (Dei voluntate I. 1. 1).

San Agustín dice que el bien creado es bueno en cuanto es orden (Thompson 2012: pp. 527-528). Por ello, la reflexión sobre el orden aparejaba también una explicación acerca del mal y de la posibilidad de considerar una conciliación entre Dios como sumo Bien y las múltiples presencias del mal. San Agustín, en este contexto, nos introduce de lleno en el tema del interiorismo: "La causa principal de este error es que el propio hombre se desconoce a sí mismo (homo sibi ipse est incognitus)" (I. 1. 3).

¿Qué significa "Conocerse" para el Hiponense? En principio, estar en tensión respecto de: a) separarse de lo sensible, recedendi a sensibus (I. 1, 3); b) replegarse en su propio espíritu, animum in seipsum colligendi (I. 1, 3) y c) vivir en contacto consigo mismo, es decir, meditar, in seipso retinendi (I. 1, 3). San Agustín propone dos terapias complementarias para alcanzar esta purificación de la vida banal o ignorancia, tan propia de la tradición (neo) platónica: por un lado, la meditación (así interpretamos solitudo, que es el término que emplea nuestro autor) y, por otro, el estudio de las artes liberales (liberalibus medicant disciplinis I. 1, 3) (Norden 1916: p. 670). Desde la dedicatoria a Cenobio, san Agustín manifiesta su convicción de que los hombres estudiosos y buenos se purifican en la medida en que se dedican al estudio. Tal purificación se hace ostensible en el tratamiento de temas arduos, como la comprensión de la realidad y del orden que en ella se expresa: la técnica del diálogo no sólo requiere una mente despierta sino también la disposición al trabajo en equipo. Sin duda, de las artes liberales, la poesía es aquella que más nítidamente refleja esta captación del mundo, pues -en especial en el marco de la preceptiva latina tardo-antigua- la antítesis es uno de sus condimentos estilísticos esenciales.

En este contexto, se explica el llamado a Zenobio, a quien dedica la obra, a amar la belleza absoluta (I. 1. 4) y a purificar el alma mediante el estudio asiduo; ya en este escrito temprano se manifiesta con fuerza la convicción agustiniana de que el mundo nunca está privado de belleza, pero los hombres que no practican la ascesis del estudio dejan de percibirla como tal. La profunda carencia de no amar la belleza implica 
necesariamente que el bien pierde fuerza de atracción, pues se diluye la evidencia de su ser; se articula así la idea de que sin belleza ni bien no es posible trazar una línea de comprensión de la realidad; en efecto, se apaga la luz del ser para quien no es capaz de gozar y entender su belleza (Sohn 2007: pp. 47-57).

Llega el final del libro I sin mayores conclusiones. Se reinicia la conversación allí donde había quedado: si Dios conduce todas las cosas por el orden y si Dios mismo se conduce mediante el orden (I, 10, 28). Estamos frente al núcleo de la concepción de orden, que se mueve en distintos planos: a) el conocimiento de las oposiciones en la realidad; b) la experiencia que el hombre puede alcanzar de sí mismo y c) los caminos de la pedagogía de Dios. Esta conjunción de elementos constituye también el lugar del sabio, en cuanto su ejercicio de comprensión lo mantiene fijo en Dios (cum Deo II. 1. 2).

La idea del sabio trae aparejado su opuesto, el necio, y una nueva reflexión sobre el orden, a partir de esta oposición: si el necio obra según un cierto orden, pierde sentido la afirmación que se había dado por segura, es decir, que Dios conduce todas las cosas mediante un cierto orden. $\mathrm{Si}$, por el contrario, el necio actúa independientemente de las relaciones que hacen posible el mundo, hay algo fuera del orden. Trigecio y Licencio intentan contrarrestar este argumento que desdice los suyos: la vida de los necios es posible por el orden previsto por Dios, por su Providencia, y no por los mismos necios. De aquí san Agustín extrae una consecuencia capital para el desarrollo del diálogo: en la sociedad, en el comportamiento de los animales y en la composición de las obras literarias es posible encontrar numerosos ejemplos de cómo elementos por sí mismos negativos desarrollan una función positiva al interior de una ensambladura y, por ello, contribuyen con su desarmonía a la armonía.

Hay dos caminos para alcanzar este conocimiento: por un lado, la formación en las artes liberales hace posible comprender la racionalidad del mundo (McWilliam 1999: p. 602); por otro lado, quien no es capaz (o no tiene la voluntad) de realizar esta tarea debe apegarse, por lo menos, a la autoridad de la fe (II. 5. 16). La filosofía salva a muy pocos, pues enseña el principio de todas las cosas que carece de principio (II. 5. 16).

En este punto, se retoma la definición de orden como el medio por el cual Dios conduce todas las cosas. A partir de aquí se plantea el tema filosóficamente más grave del diálogo: si resulta inaceptable que el mal surgió como parte de la creación, la dificultad que se presenta puede ser formulada mediante la siguiente pregunta: ¿es posible afirmar que nada sucede fuera del orden, si el acontecimiento originario del mal no puede reconducirse al orden de Dios?

La perspectiva alegórica. En I, 8, 24, San Agustín insiste en que la preocupación por el conocimiento del orden y la frecuentación de la poesía se encuentran íntimamente vinculados en quien, como Licencio, se conjuntan ambas vocaciones. Si la preocupación por el conocimiento de las Artes Liberales resulta equilibrada, nos prepara tanto moral como intelectualmente para recibir la verdad y alcanzar la beatitud eterna (McWilliam 1999: p. 602; Ramelli 2011: p. 336).

Si bien la purificación como requisito insoslayable para estar efectivamente en tensión hacia la sabiduría la encontramos decididamente en Platón (Fedón 67 a), san Agustín le 
da un contenido nuevo al considerar que aquella purificación puede cumplirse en términos de estudio. La dedicación y el esfuerzo del estudio, tal como se presenta desde su etimología, conllevan una purificación en tanto nos ponen en movimiento hacia la verdad; en este sentido, las dificultades que anota san Agustín de tantas personas de pasar de un apego difuso y melancólico al estudio (y a la investigación agregamos hoy) a efectivamente estudiar e investigar pertenecen -al parecer- a todas las épocas; el Hiponense trabaja aquí el símil médico - enfermo: todos se ponen de pie cuando escuchan las voces de vida bienaventurada o sabiduría y alzan las manos esperando recibirlas en tanto menesterosos o enfermos de distintos males (variisque morbis impeditis I, 8, 24); sin embargo, cuando deben ponerse en manos de un médico (procedimiento alegórico por "sabio") y someterse a los duros preceptos de la medicina o "sabiduría" (Brodňanská \& Koželová 2013: p. 45), casi todos eligen mantenerse en la enfermedad o "ignorancia” ( $i n$ pannos suos recidunt); en este pasaje (I, 8, 24), el vocablo latino pannus, que podemos traducir por "andrajo" o "harapo", expresa la indigencia moral en términos de ignorancia y de autocompasión (vivunt miseri, vivunt tamen I, 8, 24).

Ante este cuadro de apetencias difusas y de ausencia de voluntad para alcanzar el mandato platónico de la purificación, se expresa la confiada exhortación agustiniana a permanecer en la poesía: Vade ego interim ad illas Musas. (I, 8, 24).

Píramo y Tisbe en clave alegórica. San Agustín le propone a Licencio un ejercicio de lectura que presente a los amantes reinterpretando la misma naturaleza del relato, según -agregamos nosotros- el modelo horaciano sobre los mitos del Hades, de las observaciones de Macrobio al inicio del Somnium y de los comentarios a Virgilio en Saturnalia: ... scis quid te facere velim? (I, 8, 25).

Con esta pregunta, san Agustín introduce, en un texto sumamente breve, su propuesta de relectura; presentamos el fragmento:

“-Cuando Píramo y ella mueran, sobre el cuerpo del que apenas respira, según has de cantar, en el mismo dolor en que se encenderá tu poema con acentos más emotivos, hallarás muy excelente oportunidad. Detesta su funesta sensualidad y sus envenenados incendios, origen de aquellas desgracias; después, elévate en el canto de un amor puro y sincero, con que las almas, sostenidas por el estudio y embellecidas por la virtud, se desposan con el entendimiento por la filosofía, y no sólo evitan la muerte, sino gozan de vida dichosísima.

Aquí Licencio estuvo un rato silencioso y vacilante, y después, con la cabeza gacha, se retiró". ${ }^{7}$

La estructura de la historia de amor de Píramo y Tisbe, tal como la retrata Ovidio en Metamorfosis (IV, 55-166), puede presentarse en estos términos: los adolescentes se

7 I, 8, 25: Ubi se Pyramus et illa eius supra seminecem, ut cantaturus es, interemerint, in dolore ipso, quo tuum carmen vehementius inflammari decet, habes commodissimam opportunitatem. Arripe illius foedae libidinis et incendiorum venenatorum exsecrationem, quibus miseranda illa contingunt, deinde totus attollere in laudem puri et sinceri amoris, quo animae dotatae disciplinis et virtute formosae copulantur intellectui per philosophiam et non solum mortem fugiunt, verum etiam vita beatissima perfruuntur. Hinc ille tacitus ac diu consideratione nutans, motato capite abscessit. 
enamoraron apasionadamente. Los padres no estaban de acuerdo con estos amores e hicieron lo posible para que los jóvenes dejaran de verse. Para vivir su amor decidieron huir lejos del solar paterno. Se citaron a las afueras de la ciudad, junto a un moral y una fuente. Tisbe llegó primero. Un león bebía junto a la fuente. Tisbe huyó, perdiendo el manto en la huida. El león lo desgarró y lo manchó con la sangre de una presa reciente. Cuando llega Píramo a la escena cree que la fiera ha devorado a Tisbe; desesperado se da muerte. Tisbe decide volver sobre sus pasos: se coloca junto al moribundo y se suicida, a fin de unirse a su amado para siempre. ${ }^{8}$

Mediante la contraposición entre las indicaciones de san Agustín y la estructura del texto, tal como se presenta en Ovidio, queremos establecer ciertas pautas de comprensión. La alegoría puede existir también como un concepto previo en la mente del escritor y no solo después de la interpretación (Turner 2010: pp. 71-82).

El Hiponense ofrece un procedimiento literario que resulta, al mismo tiempo, argumento y explicación. Naturalmente ambas instancias se influyen mutualmente: en efecto hay una verdad inconmovible que transmitir y una forma literaria que, en tanto experiencia estética, llega a la plenitud conjunta de la verdad y de su expresión. Esta tensión entre el contenido y la forma (...in dolore ipso, quo tuum carmen vehementius inflammari decet, habes commodissimam opportunitatem... I, 8, 25) expresa lo que san Agustín denomina "oportunidad" de remontar el curso de los sucesos: la viva sensualidad que registra Ovidio, quien, como sabemos, no tiene intereses de edificación moral, se registra aquí como dolor que lleva a la purificación, con el cual volvemos al núcleo de la tradición neoplatónica (aquí hemos anotado el lugar del estudio en la visión agustiniana) y de la transformación de los personajes: de la pasión que los consume hasta morir por la propia mano a escalar hacia la virtud y el entendimiento por la filosofía. La palabra que para san Agustín expresa esta transformación es disciplinis, es decir mediante la aplicación al estudio, fuente de la felicidad más profunda (vita beatissima I, 8, 25).

Este procedimiento, de tan profundas resonancias en la cultura medieval y en el Renacimiento temprano, no encuentra una aceptación natural en el lector moderno; para hacerle justicia en cuanto procedimiento debemos reconstruir, como hemos intentado, la cultura que sostuvo la andadura conceptual, pues, de lo contrario, nos resultará artificial y forzado. Nos aproximaremos un poco más, si vemos a la tradición ovidiana transformarse en nuevo horizonte intelectual.

La propuesta agustiniana nos permite comprender el modo de orientar un pre-texto hacia un sentido que el escritor tardo antiguo desea ver renovado: las raíces de la comprensión de la fe, de la conducta y de la escatología, en cuanto no se limitan únicamente de la "vida del creyente" sino en cuanto a una preceptiva que la expresa y la contiene (Calabrese 2015).

8 Para un análisis completo de su significado en Ovidio vid. Spentzou (2009). 


\section{e) Conclusión}

En nuestro artículo hemos trabajado el concepto de alegoría, distinguiéndola del núcleo primordial del símbolo, como un modo de leer indisolublemente ligado a un modo de escribir; en este sentido, postulamos que el procedimiento alegórico fue una delimitación posible al modo de la permanente renovación del símbolo. En razón de lo anterior, el límite conceptual de la alegoría acompañó el complejo decurso de la cultura clásica hasta su encuentro con el cristianismo, hecho que la entronizó como una muy determinada pedagogía que puso el acento en la moralidad de los actos humanos. De los muchos ejemplos posibles tomamos una de las tantas lecturas alegorizantes del poeta Horacio, a quien san Agustín frecuentó asiduamente, como sabemos por Las Confesiones; nos resultó metodológicamente pertinente el modo en que el giro de te fabula narratur multiplica el sentido moral de la reducción alegórica del mito de Tántalo.

El joven Agustín integró aquella estructura literaria a la compleja mirada del neoplatonismo; en este contexto, el gramático Macrobio aportó elementos que permitieron fundir la búsqueda filosófica y el recurso literario en la argumentación.

Caracterizamos como una instantánea a la conjunción de todos aquellos procesos en el pasaje del De ordine, porque muestra los pasos de una preceptiva filosófico-literaria; nuestro punto de interés, sin duda, se encuentra en las indicaciones del procedimiento desde una perspectiva cristiana, cuya vitalidad se prolongó hasta el Renacimiento temprano.

El recurso de san Agustín descansa en la determinación del sentido como algo dado objetivamente y, en cuanto tal, dispuesto en textos; así se considera también que la cultura puede y debe asimilarse a una forma espiritual, a partir de la cual saber consista en disponer de una interpretación. Da el Hiponense así un primer espacio conceptual a la verdad sin hacer desaparecer la idea de multiplicidad que se instala junto a la de interpretación: la verdad se sostiene en la dimensión de lenguaje y de las obras de cultura. Para san Agustín no existe conflicto de ninguna naturaleza entre la aceptación de la verdad y su progresiva iluminación mediante el recurso de la interpretación.

Esta distancia se irá ampliando cuando las artes de gramático se apliquen a la Escritura, palabra de Dios, texto inspirado, con palabras de san Pablo (2 Tim. 3, 16). Aceptado lo anterior, la exégesis se configura sobre un texto definido y su sentido ilimitado, porque se encuentra fundado, en cuanto interpretación, sobre la lectura intertextual entre ambos Testamentos: la tensión entre la revelación completamente cumplida y una comprensión del sentido siempre en movimiento.

El posterior ejercicio agustiniano de interpretar el significado figurado sin lesionar el literal echa raíces en el procedimiento alegórico, pues introduce un nuevo lenguaje en el texto y establece, por fuera del espacio retórico en el que fue previsto y pensado, un nuevo sentido tropológico. El desafío radical de la alegoría en sede literaria consiste en que el autor de turno tenga la habilidad suficiente para fusionar, como en un crisol de materia y forma, los intereses retóricos y filosóficos. Por esta razón, la hermenéutica de signo agustiniano resulta una fuerte toma de conciencia del carácter universal, progresivo y no limitado de la interpretación. 


\section{Bibliografía}

\section{Fuentes primarias}

Armisen-Marchetti, M. (Ed.). (2001). Macrobe: Commentaire au songe de Scipion. Livre I (Texte établi, traduit et commenté). Paris: Les Belles Lettres.

Armisen-Marchetti, M. (Ed.). (2003). Macrobe: Commentaire au songe de Scipion. Livre II (Texte établi, traduit et commenté). Paris: Les Belles Lettres.

Brehier, E. (Ed.). (1924-1938). Plotin: Les Ennéades (Texte établi, traduit et commenté). Paris: Les Belles Lettres.

Fedeli, P. (Ed.). (1994). Q. Orazio Flacco: Le Opere, Vol. 2: Le Satire. Roma: Istituto Poligrafico dello Stato.

Rivaud, A. (Ed.). (1925). Platon: Timée. CEuvres Complètes X. Paris: Les Belles Lettres.

Rouse, W. H. D. (Ed.). (2014). Titus Lucretius Carus: De rerum natura (The Loeb classical library). Cambridge, Mass.: Harvard University Press.

Rowe, C. J. (Ed.). (1993). Plato: Phaedo. Cambridge: Cambridge University Press.

Ruiz de Elvira, A. (Ed.). (2002). P. Ovidio Nasón: Metamorfosis, Vol. 1: Lib. I-IV. Madrid: Consejo Superior de Investigaciones Científicas.

Trelenberg, J. (2009). Augustins Schrift De ordine. Tübingen: Mohr Siebeck.

Waterfield, R. A. H. (Transl.). (2008). Plato: Republic. Oxford: Oxford University Press.

Wicksteed, P. H., \& Cornford, F. M. (Eds.). (1957). Aristotle: Physics, Vol. I: Books 1-4 (Loeb classical library). Cambridge, Mass.: Harvard University Press.

\section{Fuentes secundarias}

Bermon, E. (2011). Le "Songe de Scipion" dans la correspondance entre saint Augustin et Nectarius de Calama (Ep. 90-91; 103-104). Les Études philosophiques, 4, 521-542.

Brodňanská, E., \& Koželová, A. (2013). Alegoría médica en la poesía moral de Gregorio Nacianceno. Graeco-Latina Brunensia, 18(2), 43-66.

Calabrese, C. C. (2015). Los supuestos hermenéuticos de Agustín de Hipona. Desentrañar la palabra y transmitir su misterio. Espiritu, 64(150), 227-243.

Curtius, E. R. (1990). European Literature and the Latin Middle Age. New Jersey: Princeton University Press.

Eliade, M. (1987). The Sacred and the Profane. The Nature of the Religion. New York - London: Harcourt, Inc.

Esparza Urzúa, G. A. (2017). La formación simbólica de la cultura de la paz. Mito y política en Ernst Cassirer. In E. Junco, C. Calabrese, \& F. García Costa (Eds.), Los humanismos y la cultura para la paz. Zacatecas: Texere Editores.

Flamant, J. (1977). Macrobe et le Néoplatonisme latin à la fin du IVe siècle. Leiden: Brill.

Gersh, S. (2012). The First Principles of Latin Neoplatonism: Augustine, Macrobius, Boethius. Vivarium, 50(2), 113-138. 
Herren, M. (2017). The Anatomy of Myth. The Art of Interpretation from the Presocratics to the Church Fathers. New York: Oxford University Press.

Junco, E. (2016). Euripides y la belleza del bien. México: Texere.

Lamberton, R. (1986). Homer the Theologian: Neoplatonist Allegorical Reading and the Growth of the Epic Tradition (The transformation of the classical heritage, 9). Berkeley - Los Angeles - London: University of California Press.

Lewis, C. S. (2013). The Allegory of Love. A Study in Medieval Tradition (la primera edición es de 1936). New York: Cambridge University Press.

McWilliam, J. (1999). De ordine. In A. D. Fitzgerald et al. (Ed.), Augustine through the Ages. An Encyclopedia. Grand Rapids, Michigan - Cambridge, U. K.: William B. Eerdmans Publishing Company.

Norden, E. (1916). Vergilius Maro, Aeneis Buch VI. Leipzig: Teubner.

Pépin, J. (1976). Mythe et allégorie. Les origines grecques et les contestations judéo-chrétiennes. Paris: Études Augustiniennes.

Pfeiffer, R. (1968). History of Classical Scholarship from the Beginnings to the End of the Hellenistic Age. Oxford: Clarendon Press.

Ramelli, I. (2011). The Philosophical Stance of Allegory in Stoicism and its Reception in Platonism, Pagan and Christian: Origen in Dialogue with the Stoics and Plato. International Journal of the Classical Tradition, 18(3), 335-371.

Ricoeur, P. (1965). De l'interprétation: Essai sur Freud. Paris: Éditions du Seuil.

Setaioli, A. (2004). Interpretazioni stoiche ed epicuree in Servio e la tradizione dell'esegesi filosofica del mito e dei poeti a Roma (Comuto, Seneca, Filodemo). International Journal of the Classical Tradition, 11(1), 3-46.

Sohn, H. (2007). The Beauty of Hell? Augustine's Aesthetic Theodicy and Its Critics. Theology Today, 64, 47-57.

Spentzou, E. (2009). Theorizing Ovid; Readers and Illusions: The Imaginary and the Real. In P. E. Knox, A Companion to Ovid (pp. 389-393). New Jersey: Wiley-Blackwell Publishing Company.

Stevens, J. (2006). The Imagery of Cicero's Somnium Scipionis. In C. Deroux (Ed.), Studies in Latin Literature and Roman History (Vol. XIII; pp.155-165). Bruxelles: Collection Latomus.

Struck, P. T. (2004). Birth of the Symbol. Ancient Readers at the Limits of Their Texts. Princeton, NJ Oxford: Princeton University Press.

Thompson, S. E. (2012). What Goodness Is: Order as Imitation of Unity in Augustine. The Review of Metaphysics, 65(3), 525-553.

Turner, D. (2010). Allegory in Christian Late Antiquity. In R. Copeland, \& P. Struck, The Cambridge Companion to Allegory (pp. 71-82). Cambridge: Cambridge University Press.

\section{Resumen}

En el presente artículo pretendemos establecer una relación entre mito y alegoría según el tratamiento de la tradición literaria helenístico-cristiana, para considerar la aplicación de la alegoría con función filosófica y su recepción por san Agustín. Ejemplificamos el proceso con la 
Claudio César Calabrese

Alegoría, mito y artes liberales en san Agustín De ordine I. 8. 24

interpretación agustiniana de la narración de Píramo y Tisbe según De Ordine. Concluimos en el alcance argumentativo de la alegoría para fundar una preceptiva filosófica.

\section{Palabras clave}

San Agustín; alegoría; orden; artes liberales; hermenéutica

Dr. Claudio César Calabrese / ccalabrese@up.edu.mx

Department of Humanities

Universidad Panamericana, Campus Aguascalientes

Josemaría Escrivá de Balaguer N 101, CP 20290 Aguascalientes, Mexico 